\title{
Motile Human Sperm Sorting by an Integrated Microfluidic System
}

Hong-Yuan Huang ${ }^{1,2}$, Hui-Ting Fư ${ }^{3}$, Hsin-Yao Tsing ${ }^{3}$, Hung-Ju Huang ${ }^{2}$, Chin-Jung Li' and Da-Jeng Yao ${ }^{3 *}$

${ }^{1}$ Department of Obstetrics and Gynecology, Chang Gung Memorial Hospital, Kwei-Shan, Tao-Yuan, Taiwan

${ }^{2}$ Department of Obstetrics and Gynecology, Chang Gung University and College of Medicine, Kwei-Shan, Tao-Yuan, Taiwan

${ }^{3}$ Institute of NanoEngineering and MicroSystems, National Tsing Hua University, Hsinchu, Taiwan

\begin{abstract}
The integrated microfluidic system consists of laminar stream-based microchannels and flow cytometric analysis to enhance the human sperm motility sorting efficiency. This modified sperm sorter provided a new selection that had advances in resolving traditional sorting problems. In order to design the sorting sorting device, CFDRC simulation was performed to analyze the phenomenon of 3-D flow field. Microscopic examination revealed that sperms with different motility. For the purpose of confirming the real sorting situation, the proportions of living and dead sperm after sorting were readily identified and quantified through use of flow cytometric analysis. The viability results of sample injected from two-outlet and four-outlet chip were $95.2 \%$ and $92.2 \%$ that presented the higher quality of sorting sperm. As a result, more amounts of living sperms that mean motile sperms were counted in four-outlet channels. With this approach, the characteristics of laminar flow and a novel design were utilized to demonstrate the microfluidic system with high efficiency for sperm motility sorting. Therefore, the optimized microfluidic system provides a good opportunity to use and an inexpensive requirement to select the most appropriate sperms before IVF (in vitro fertilization) process.
\end{abstract}

Keywords: Microfluidic chip; Motile sperm; Sorting; Laminar flow; Long-term stability

\section{Introduction}

With the development of Micro Electro Mechanical System (MEMS) technologies in medical applications, numerous experiences are now available that may enable to actually apply in a microfluidic system [1]. Microfluidics have been widely applied to biomedical fields [2-8], because control of fluid transport is useful for cell analysis systems, drug delivery systems, and assisted reproductive technologies. Microfluidic technology has been utilized in numerous biological applications specifically for miniaturization and simplification of laboratory techniques [9]. Reproductive medicine has played an important role in the field of medical science. The main reasons of infertility from male are the abnormality and deficient amount of sperms. These problems could be solved by the assisted reproductive technology (ART) and the typical solutions are the in vitro fertilization (IVF) technology and intracytoplasmic sperm injection (ICSI) [10,11]. However, traditional sperm sorting methods, such as sperm washing that is an easy way to select live sperms by once or twice centrifugation, swim-up techniques that is the most common method used in IVF procedure, the motile sperms were separated by the capability to swim out of the semen [12-14], density gradient centrifugation (DGC) that the motile sperms were selected by the density discontinuous gradient, sperm migration that is based on spermatozoa self-propelled activity, sperm sedimentation that Tea et al. proposed which combined swimup with the sedimentation process and centrifugation, often require manual selection, and time consuming. Besides, the probability of DNA damage is increased via these ways [15-17]. Among the sperm sorting via microfluidic devices, Horsman et al. [18] applied a microfabricated device based on the ability of motile sperm to move out of their initial streamlines in the laminar fluid stream, Cho et al. [19] proposed a horizontally-setup gravity-driven pumping system, which greatly simplifies the sorting step without centrifugation method, the purity of motile sperm after separating was nearly $100 \%$. Nowadays, there are some innovative sperm separation have been developed. For example, Lin et al. [20] proposed a microfluidic device with diffuser chamber, which separate the sperm by the velocity gradient. Ainsworth et al. [21] proposed an electrophoretic system to isolate the human spermatozoa by size and electric field, which is rapid, safe and low level of DNA damage. In particular, the channels and reservoirs were coated with $1 \%$ Bovine Serum Albumin (BSA) to improve the surface hydrophilicity [22]. By varying the channel dimensions, numerical simulations were applied to confirm the separation efficiency of sperm sorter. The device isolates motile sperm from immotile sperm and other cellular debris [23]. Microfluidic devices were fabricated from poly (dimethylsiloxane) (PDMS) offer the ability to improve biological and medical capabilities. Wu et al. [24] described a PDMS-based sperm sorting device, which has been surface-modified by coating PEG-MA to create a moderately hydrophilic and non-fouling surface [24]. As the demonstration from our previous study [25], the microfluidic system can effectively separate raw semen samples into immobile and mobile groups without centrifugation steps. Simultaneously, the damage to the sperms could be avoided. In addition, the microfluidic device can reduce the time required for intracytoplasmic sperm injection (ICSI) treatment that is used to increase sperm concentration in poor-quality semen samples [10]. The results suggest that this device may be clinically useful for ICSI treatment in human assisted reproductive technology. However, no method from the above studies was obtained to confirm the sperm quantity test that can readily evaluate motility information. By fluorescent stain [26] and statistics method, such as flow cytometric analysis [27], the amount of sperms included living and dead sperm can be characterized, and a statistic model for analyzing the sorting efficiency of motile sperms was developed. The concept of mouse sperm isolation by microfluidic system has been proved [28].

In this study, the integrated microfluidic system which consists of

*Corresponding author: Da-Jeng Yao, Institute of Nano Engineering and MicroSystems, National Tsing Hua University, No 101, Sec. 2, Kuang-Fu Road Hsinchu 30013, Taiwan, Tel: +886-3-5715131 ext. 42850; Fax: +886-3-5745454; E-mail: djyao@mx.nthu.edu.tw

Received April 14, 2014; Accepted May 20, 2014; Published May 25, 2014

Citation: Huang HY, Fu HT, Tsing HY, Huang HJ, Li CJ, et al. (2014) Motile Human Sperm Sorting by an Integrated Microfluidic System. J Nanomed Nanotechnol 5: 199. doi:10.4172/2157-7439.1000199

Copyright: ( 2014 Huang HY, et al. This is an open-access article distributed under the terms of the Creative Commons Attribution License, which permits unrestricted use, distribution, and reproduction in any medium, provided the original author and source are credited. 
laminar stream-based microchannels demonstrated the separation of human motile sperms, and flow cytometric analysis was used to enhance the sperm motility sorting efficiency. Considering the characteristic of the microfluidic channel, it is similar to the natural environment of the fallopian. The interactions between laminar flow and sperm activity were tested as well. Due to the laminar flow in different directions, the semen sample was loaded into a straight laminar flow and the results showed that most motile sperms with lateral activity could swim across the two fluid boundaries to be sorted. The working concept lies in the design of multiple channel systems that control the formation of multiple laminar streams, and motile sperms could swim across the stream lines and were collected by the dedicated reservoirs. In order to increase the sorting efficiency of the sorting device, CFDRC simulation was performed to analyze 3-D flow field to obtain the optimal design. Microscopic examination revealed that sperms with different motility. The fluorescence could be measured simultaneously to identify living sperm differed from dead ones by using flow cytometry. For the purpose of confirming the real sorting situation, the proportions of living and dead sperm after sorting were readily identified and quantified through use of flow cytometric analysis. Moreover, the microfluidic system

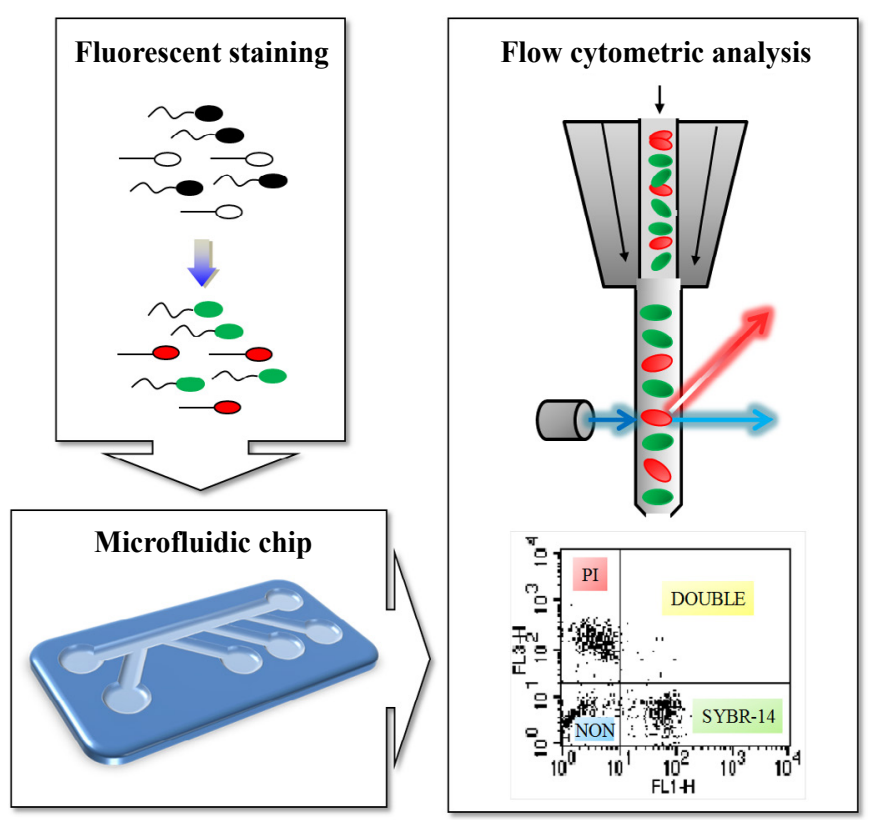

Figure 1: Schematic illustration of sorting process for microfluidic system.

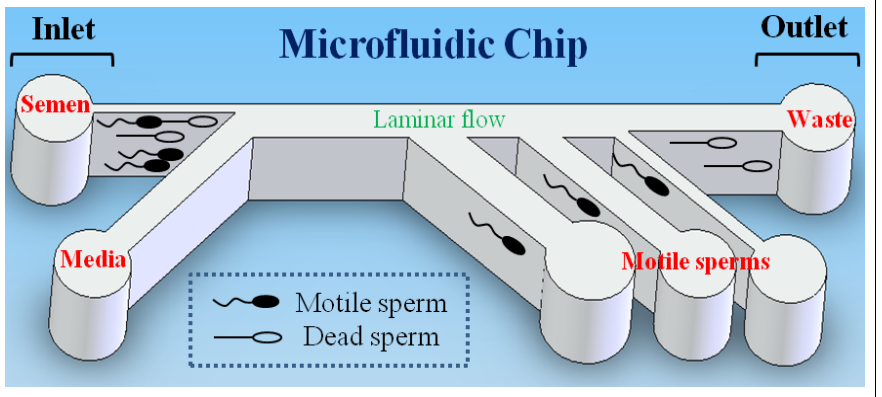

Figure 2: The schematics of multiple channel microfluidic chip. The motile sperms would be sorted either with random swimming direction by laminar flow. enhances the sorting efficiency in the quality and quantity of sperm. The modified surface of flow channel improves long-term stability of the bio-chip. With this approach, the characteristics of laminar flow and a novel design were utilized to demonstrate the microfluidic system with high efficiency for sperm motility sorting.

\section{Design and Methods}

\section{Microfluidic system}

A schematic illustration of sperm sorting process for microfluidic system is shown in Figure 1. Briefly, semen samples were first stained by fluorescent dye and revealed that living sperm differed from dead sperm. After injecting the stained sperms into microfluidic chip from inlet reservoir, the results could be recorded by microscope. Microscopic examination revealed that sperms with different motility. Finally, the proportions of living and dead sperm after sorting were readily quantified through use of flow cytometric analysis.

The study is divided into two kinds of sample injection method. One is gravity-driven. The other is pumping system by syringe pump. At first, the method of gravity-driven was utilized to confirm the feasibility of the chip. Rear section, the syringe pump injected the sample into our chip. It could control the flow rate accurately. Therefore, the flow velocity was slower and stable. Decreasing the flow velocity provides more opportunities of sorting sperm. Using controlled microfluidic system to enhance the sperm motility sorting efficiency.

\section{Design of microfluidic chip}

The purpose of this research was to sort motile sperms based on the effect of laminar flow in multiple channel and the moving activity of sperm samples. The ideal of multiple channel microfluidic chip was presented here, as shown in Figure 2, and the efficiency of sorting sperm motility could be increased by the suitable design of the microchannel.

For the purpose of increasing the sorting efficiency of the microfluidic chip, CFDRC simulation was performed to analyze 3-D flow field to obtain the optimal design. The CFD-ACE+ 2006 of CFD commercial software was utilized. The flow module and two-fluidic were used at steady state to solve for flow of two-fluidic system which contains two continuous with independent properties. Viscosity and density of semen sample and of HEPES medium were assumed to be constant. The value of viscosity of semen sample and HEPES medium were assumed to be $0.00089 \mathrm{kgm}^{-1} \mathrm{~s}^{-1}$ and $0.0007 \mathrm{kgm}^{-1} \mathrm{~s}^{-1}$ at $28^{\circ} \mathrm{C}$. The density was assumed to be the same as water $997 \mathrm{kgm}^{-3}$. The special value of "Alpha" was set up. The value of Alpha of semen sample and HEPES medium were assumed to be 1 and 0 . Therefore, the simulation result of concentration can indicate by value of Alpha. The result showed that ratio of flow rates was most suitable at 1:5, as shown in Figure 3. It means that dead sperm and impurities stay original streamline, and the motile sperm moved from their initial streamlines to HEPES medium whose Alpha is 0 . According to the simulation analysis, it is clarified that sorting mechanisms of the chip with multiple channels would improve efficiency of sorting results.

According to the simulation results, the length and the width of the main microchannel have been determined to be $5000 \mu \mathrm{m}$ and $400 \mu \mathrm{m}$, respectively. The height of the microchannel is $70 \mu \mathrm{m}$. Semen sample was employed in the experiment, and the results were observed by using the microscope.

\section{Calculation of flow velocity}

Velocity of sperm is common at $70 \mu \mathrm{m} / \mathrm{s}$. We present a control 


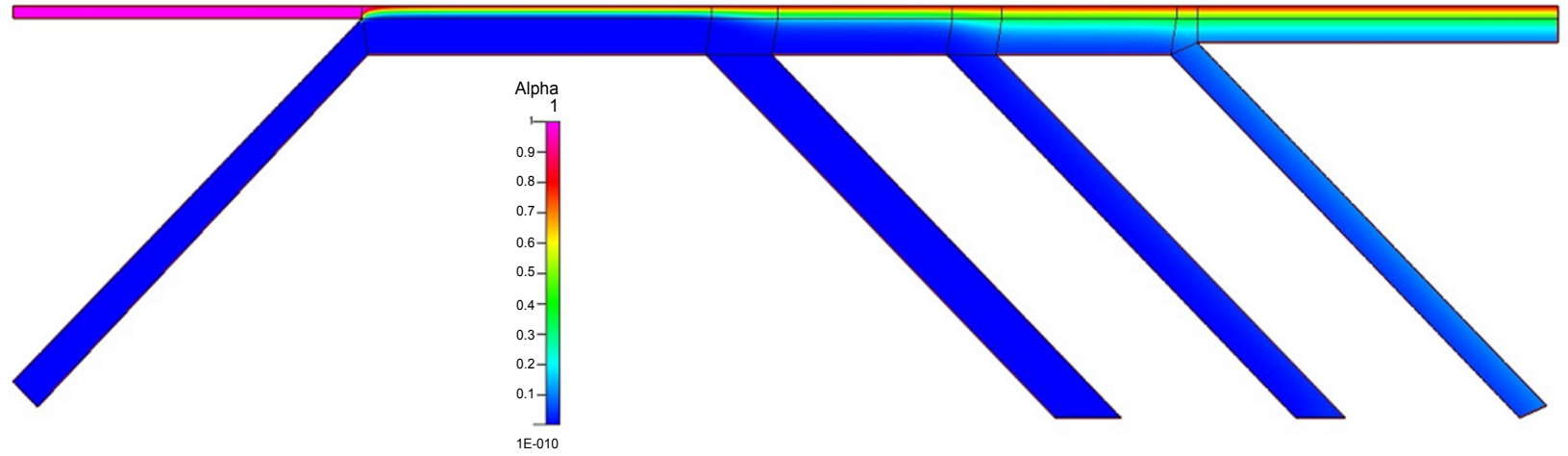

Figure 3: The result of simulation of concentration showed that ratio of flow rates was most suitable at 1:5

microfluidic system to change the flow rate. This method was different from the gravity-driven. It used syringe pumps to change the flow rate. Decreasing the flow velocity provides more opportunities of sorting sperm. The unit of setup of syringe pumps is $\mathrm{Q}(\mu \mathrm{l} / \mathrm{min})$. By the hydrodynamics, flow velocity is associated to flow rate and crosssection area of microchannel. The simulation result showed that ratio of flow rates was most suitable at 1:5. Therefore, the flow rate of pumps of semen and HEPES medium were set $0.1 \mu \mathrm{l} / \mathrm{min}$ and $0.5 \mu \mathrm{l} / \mathrm{min}$ individually. According to the results of calculation, the flow velocity of inlet is about $350 \mu \mathrm{m} / \mathrm{s}$, and the outlet is about $70 \mu \mathrm{m} / \mathrm{s}$ due to multioutlet dispersed flow. Normally, the average moving velocity of motile sperm is about $70 \mu \mathrm{m} / \mathrm{s}$. Therefore, the flow velocity of the sorting channel was set $70 \mu \mathrm{m} / \mathrm{s}$. As a result, there are only motile sperm could be separated and counted. The slower flow velocity is conducive to sorting sperm, even increases the sorting efficiency.

\section{Fabrication}

A standard soft lithography technique is modified to fabricate microfluidic channels using polydimethylsiloxane (PDMS). Briefly, the microfluidic chip employed in this study was made of the SU-8 photoresist on silicon wafer for $50 \mu \mathrm{m}$ deep as master mold to form microchannels. Subsequently, Slygard 184 and curing agent were mixed at a 10:1 then poured on top of the master mold and degassed in vacuum, and finally cured in an oven at $90^{\circ} \mathrm{C}$ for one hour to obtain the PDMS microchannels. The resulting PDMS channels were cut off from the reservoirs to connect the channels, and PDMS device was treated for thirty seconds with oxygen plasma then bonded with clear glass to form the separation microchannel. In addition, poly (ethylene glycol) methyl ether methacrylate (PEG-MA) (MW 475) is hydrophilic and biocompatibility. PEG-MA coated PDMS microchannels were fabricated for microfluidic system and for demonstrating the hydrophilicity of this bio-chip. Before experiment, the microchannels and reservoirs must be coated with 1\% BSA to enhance its hydrophilic and reduce cell nonspecific absorption.

\section{Surface modification}

Hydrophilic surfaces on polydimethylsiloxane (PDMS) are desirable for microfluidic channels. A novel method for creating stable and hydrophilic surface in which PEG-MA is bonded with PDMS was developed. The surface characterization of PEG-MA coated PDMS was performed by using contact angle measurements to determine hydrophilicity and long-term stability. The untreated PDMS is very hydrophobic and the effect of $\mathrm{O}_{2}$ plasma treatment is temporary hydrophilicity. After 3 days, the contact angle result of $\mathrm{O}_{2}$ plasma treatment is $100.13^{\circ}$. The result of PEG-MA coated hydrophilic surface have a contact angle of $35.97^{\circ}$. Additionally, PEG-MA coated PDMS exhibits long-term stability maintaining for at least 3 days. The modified surface allows for development of microfluidic system that is portable and reliable to operate.

\section{Results}

\section{Material and preparation}

Human semen samples were obtained from men undergoing evaluation for infertility after a minimum of 3 days of abstinence after informed consent and Chang Gung Memorial Hospital IRB approval (CGIRB\#97-1641B/101-3687B). After liquefaction, a semen analysis was performed by a single individual and consisted of assessment of semen volume, $\mathrm{pH}$, viscosity, liquefaction, sperm count, sperm motility, sperm agglutination, strict sperm morphology and cell contamination. Semen samples containing spermatozoa with forward progression were selected for experimental use according to the WHO guideline of human semen parameters (grade a and b motility sperm $>50 \%$ or only grade a motility sperm. $>25 \%$ ). There are four grades about sperm motility. It fast forward motion and doesn't see the tail movement called "grade a" motility. It forward motion and clearly see the tail movement called "grade b". It walks around in circles called "grade c". And then, it no moving phenomenon called "grade d". Therefore, the design concept of our study can select out sperm which is grade a and b. In order to avoid the activity decline of obtained sperms, the experiment will be carried out as soon as possible. Subsequently, keeping the sample still for a while, the supernatant of sample was used in this experiment. The advantage of this method was easy to use to get a low impurity samples, and to eliminate the need for complex process, in addition, the sample can be avoided the damage which was caused by centrifugation steps.

\section{Fluorescent staining}

Human semen was employed in this study and observed through the microscope. The swimming direction of sperms indicate that all kinds of sperms either with random, or combined motility can be selected successfully in the desired reservoir. The recoded images from the inlet intersection of two streams were revealed that motile sperms passed through the straight-curve flow boundary into the curve laminar flow, as Figure 4a showed. In addition, the semen samples were loaded into a curve laminar flow through straight-curve stream and it would offer a y component force for motile and dead sperms to be selected, as shown in Figure $4 \mathrm{~b}$. Due to the laminar flow in different directions, the sperms injected from a curve inlet would offer a y component force for 

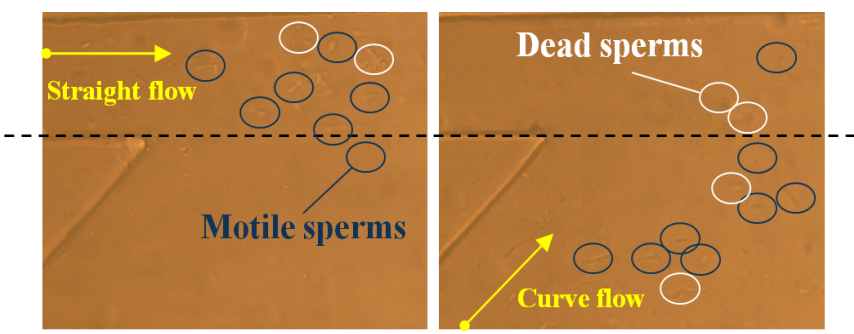

Figure 4: The recoded images were showed at the inlet intersection of two streams. (a) The motile sperms passed through the straight-curve flow boundary into the curve laminar flow. (b) The motile and dead sperms were sorted while semen sample was loaded into a curve laminar flow through straight-curve stream and it would offer a y component force.
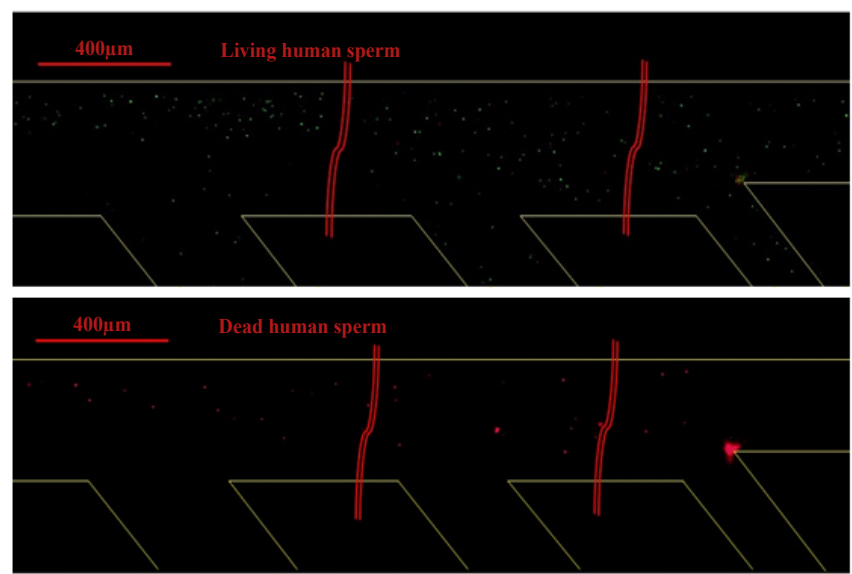

Figure 5: At the first outlet-down, the number of collection of motile sperm was less due to the faster flow velocity. And then, the multi-outlet dispersed flow let flow velocity slower at second and third outlet-down. Therefore, the more motile sperm was collected there. (a) The tracking picture of the motile sperm collected during 1.38 second. (b) The dead sperm separation. (Blue circle: the motile sperm collected).

motile and dead sperms to be close to the flow boundary. As a result, the dead sperms were easier to across into outlet reservoir. On the other hand, the semen sample was loaded into a straight laminar flow and the results showed that most of motile sperms with lateral activity could swim across the two fluid boundaries to be sorted.

The living sperm in semen samples exhibited fluorescence of bright green in the nucleus stained with SYBR-14 and excited at $518 \mathrm{~nm}$. With the addition of PI, the dead sperm DNA stained bright red. The staining of sperm with combination of SYBR-14 and PI was observed by fluorescence microscope immediately. However, the fluorescent stain might affect the motility of the human sperms. According to the dyeing process of our experiments, we found that the amount of SYBR-14 added will affect the motility of the human sperm. Although the staining effect is very obvious that the concentration of SYVR-14 reaches to $5 \mu \mathrm{l}$, it critically damages the sperm motility. We decided to control the concentration of SYVR-14 down to $0.5 \mu \mathrm{l}$, even the intensity of green fluorescence will be weaker. The concentration of SYVR-14 is good for the human sperm to keep the motility. Moreover, after injecting the semen into the microfluidic chip from inlet reservoir, and the results could be recorded by the video of microscope. The motile sperm could be collected at three outlet-down. The images of experiment could be seen that semen laminar flow lines are wider near the outlet. It was same as result of simulation. At the first outlet-down, the number of collection of motile sperm was less due to the faster flow velocity. And then, the multi-outlet dispersed flow let flow velocity slower at second and third outlet-down. Therefore, the more motile sperm was collected there, as shown in Figure 5. This phenomenon would indicate that the quality and quantity of sperm sorting was increased.

\section{Flow cytometric analysis}

The sperms were stained with SYBR-14 (green fluorescence) and PI (red fluorescence) to assess the differences in sperm viability and motility among human semen. The results showed that the proportions of living and dead sperm in human semen could be identified via the use of staining with fluorescent dye and quantified through use of flow cytometric analysis. Scattergram dot plots showed typical flow cytometric analysis for sperm that were stained with both SYBR-14 and PI. Through the use of adjusting the experimental parameters, the initial sperm populations are located in the LL region. In addition, the living and dead sperm are located in the LR and UL region as shown in Figure 6. In Figure 7, dot-plot scattergrams were generated for semen sample from microfluidic chips with different design. The logs of green and red fluorescence for living and dead sperm are provided
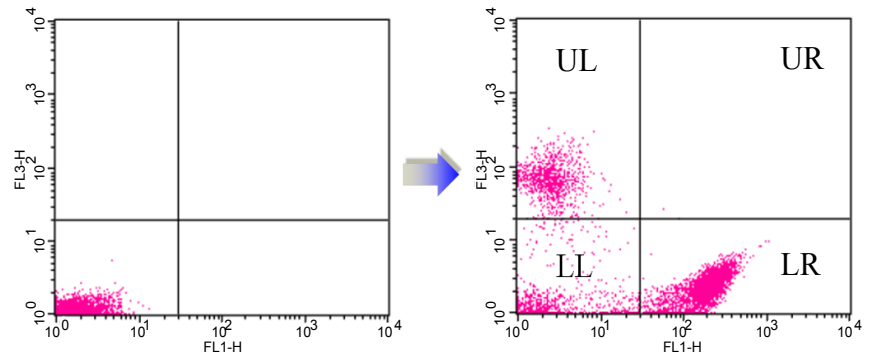

Figure 6: Scattergram dot plots showed typical flow cytometric analysis for stained sperm that were used to distinguish the living(LR region) and dead(UL region) sperms.

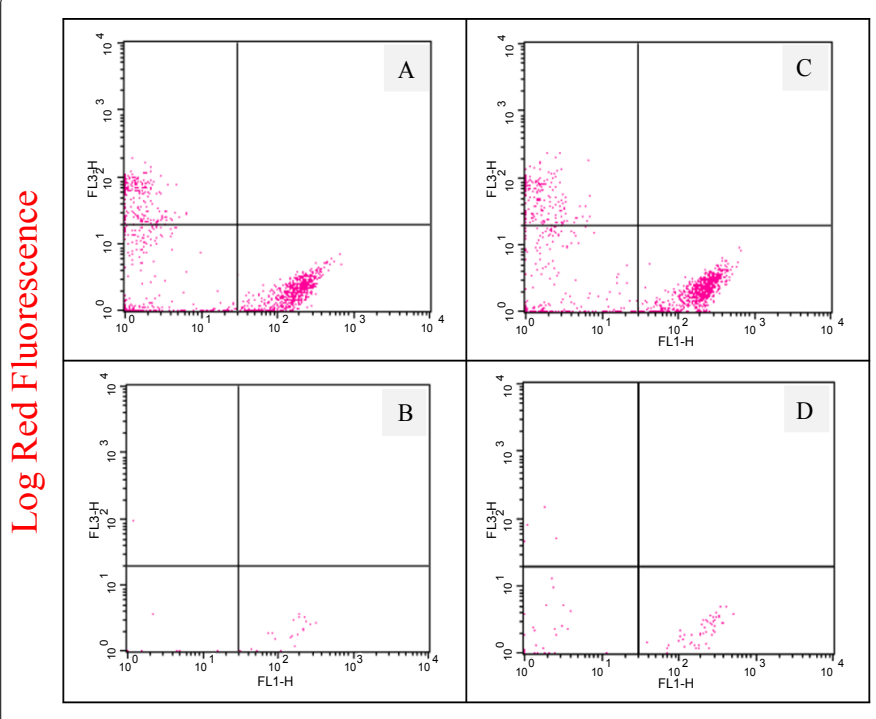

Log Green Fluorescence

Figure 7: Dot-plot scattergrams were generated for the (a) and (b) that double stained sperm injected from two-outlet microchannels, which revealed sample without sorting and after sorting. In addition, (c) and (d) were showed that double stained sperm injected from four-outlet microchannels, which revealed sample without sorting and after sorting. 


\begin{tabular}{|c|c|c|c|}
\hline Human semen & Living sperm & Dead sperm & Viability \\
\hline Original sample & 3354 & 1047 & $76.21 \%$ \\
\hline 2inlet-2outlet (A) & 647 & 215 & $75.06 \%$ \\
\hline 2inlet-2outlet (B) & 20 & 1 & $95.24 \%$ \\
\hline 2inlet-4outlet (C) & 864 & 189 & $82.05 \%$ \\
\hline 2inlet-4outlet (D) & 47 & 4 & $92.16 \%$ \\
\hline
\end{tabular}

Table 1: Sperm counts and ratio of sorting.

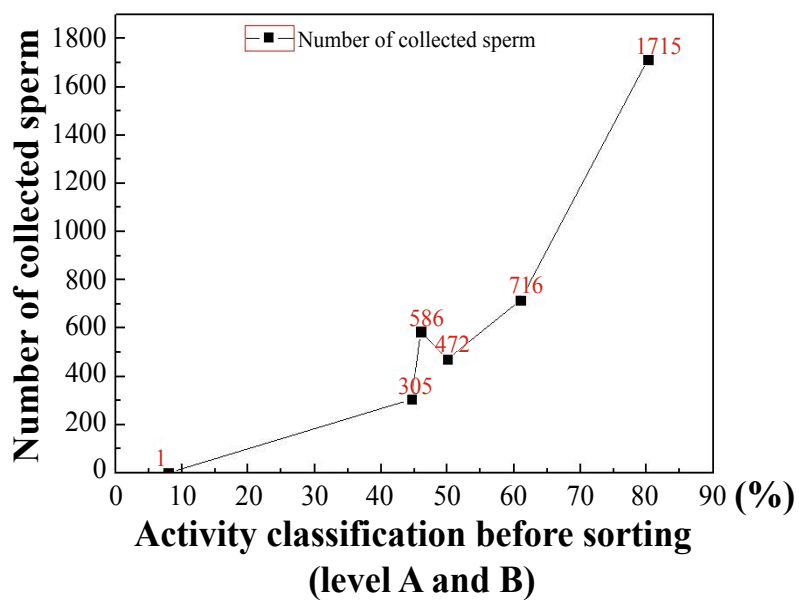

Figure 8: The number of collection of motile sperm was associated to activity classification of sperm before experiment. The Level A and Level B of activity classification were proportional to the collection of motile sperm.

along with the analysis showing the mean proportion of SYBR-14 and PI. Figure $7 \mathrm{a}$ and $\mathrm{b}$ showed that double stained sperm injected from two-outlet microchannels, which revealed sample without sorting and after sorting. In addition, double stained sperm injected from fouroutlet microchannels, which revealed sample without sorting and after sorting as Figure $7 \mathrm{c}$ and $\mathrm{d}$. Table 1 showed the sperm counts and ratio of sorting using two-outlet and four-outlet channels of microfluidic system. The viability results of original sample were $76.21 \%$ that similar with the expected results of sample injected from two-outlet and four-outlet chip which without sorting were $75.06 \%$ and $82.05 \%$. The viability results of sample injected from two-outlet and four-outlet chip which after sorting were $95.2 \%$ and $92.2 \%$ that presented the higher quality of sorting sperm. However, there are two more outlets for sorting motile sperm in the four-outlet chip. As a result, more amounts of living sperms that mean motile sperms were counted in four-outlet channels. The counted ratio of living sperms revealed the high quantity and efficiency of sorting sperm.

On the other hand, we controlled the flow rate to repeat experiments. The syringe pumps that let flow rate slower and stable was utilized to inject semen. The different situations semen was utilized to do our experiments. The results show that number of collection of motile sperm was associated to activity classification of sperm before experiment. The sperms with grade $a$ and $b$ motility of activity classification were proportional to the collection of motile sperm, as shown in Figure 8. The results demonstrated to confirm the feasibility of the chip, although human sperm which is large individual differences.

\section{Conclusion}

The integrated microfluidic system used for human motile sperm separation has been demonstrated, that consists of laminar stream-based microchannels design and flow cytometric analysis. Compared with the traditional sorting technique, the developed microfluidic system with multiple channels improves the sorting efficiency in the quality and quantity of sperm. The viability results of sample injected from twooutlet and four-outlet chip were $95.2 \%$ and $92.2 \%$ that presented the higher quality of sorting sperm. As a result, more amounts of living sperms that mean motile sperms were counted in four-outlet channels, which proved more outlets can be used if quantity of live sperms should be increased. Therefore, the optimized microfluidic system provides a good opportunity to sort the human motile sperms before IVF (in vitro fertilization) process.

\section{Acknowledgment}

The authors would like to acknowledge the financial support provided by NTHU/CGMH collaboration funds (CMRPG361081, CMRPG380121 and CMRPG390441), and part of fund comes from National Science Council of Taiwan (NSC 101-2120-M-002-017, NSC 101-2314-B-182-041, and NSC 101-2221-E-007099-MY3).

\section{References}

1. Seo DB, Agea Y, Feng ZC, Critser JK (2007) Development of sorting, aligning and orienting motile sperm using microfluidic device operated by hydrostatic pressure. Microfluidic Nanofluid 3: 561-570.

2. Tomlinson AJ (1995) Enhancement of concentration limits of detection in CE and GEMS: A review of on-line sample extraction, cleanup, analyte preconcentration, and microreactor technology. Journal of Capillary Electrophoresis 2: 247-266.

3. Brody JP, Yager Y (1997) Diffusion-based extraction in a microfabricated device. Sensors and Actuators a-Physical 58: 13-18.

4. Kenis PJ, Ismagilov RF, Whitesides GM (1999) Microfabrication inside capillaries using multiphase laminar flow patterning Science 285: 83-85.

5. Weigl BH, Yager P (1999) Microfluidics - Microfluidic diffusion-based separation and detection. Science 283: 346-347.

6. Takayama S, Ostuni E, LeDuc P, Naruse K, Ingber DE, et al. (2001) Subcellular positioning of small molecules. Nature 411: 1016.

7. Ku PH, Hsiao CY, Chen MJ, Lin TH, Li YT, et al. (2012) Polymer/Ordered mesoporous carbon nanocomposite platelets as superior sensing materials for gas detection with surface acoustic wave devices. Langmuir 28: 11639-11645.

8. Hao HC (2010) A Portable Electronic Nose Based on Bio-Chemical Surface Acoustic Wave (SAW) Array with Multiplexed Oscillator and Readou Electronics. Sensors and Actuators B Chemical 146: 545-553.

9. Suh RS, Zhu X, Phadke N, Ohl DA, Takayama S, et al. (2006) IVF within microfluidic channels requires lower total numbers and lower concentrations of sperm. Hum Reprod 21: 477-483.

10. Bonduelle M, Camus M, De Vos A, Staessen C, Tournaye H, et al. (1999) Seven years of intracytoplasmic sperm injection and follow-up of 1987 subsequent children. Hum Reprod 14 Suppl 1: 243-264.

11. Devroey P, Van Steirteghem A (2004) A review of ten years experience of ICSI. Hum Reprod Update 10: 19-28

12. Englert Y, Van den Bergh M, Rodesch C, Bertrand E, Biramane J, et al. (1992) Comparative auto-controlled study between swim-up and Percoll preparation of fresh semen samples for in-vitro fertilization. Hum Reprod 7: 399-402.

13. Smith S, Hosid S, Scott L (1995) Use of postseparation sperm parameters to determine the method of choice for sperm preparation for assisted reproductive technology. Fertil Steril 63: 591-597.

4. Ricci G, Perticarari S, Boscolo R, Montico M, Guaschino S, et al. (2009) Semen preparation methods and sperm apoptosis: swim-up versus gradient-density centrifugation technique. Fertil Steril 91: 632-638.

15. Henkel RR, Schill WB (2003) Sperm preparation for ART. Reprod Bio Endocrinol 1: 108.

16. Aitken RJ, Clarkson JS (1988) Significance of reactive oxygen species and antioxidants in defining the efficacy of sperm preparation techniques. J Androl 9: 367-376.

17. Zini A, Finelli A, Phang D, Jarvi K (2000) Influence of semen processing technique on human sperm DNA integrity. Urology 56: 1081-1084. 
Citation: Huang HY, Fu HT, Tsing HY, Huang HJ, Li CJ, et al. (2014) Motile Human Sperm Sorting by an Integrated Microfluidic System. J Nanomed Nanotechnol 5: 199. doi:10.4172/2157-7439.1000199

18. Horsman KM, Barker SL, Ferrance JP, Forrest KA, Koen KA, et al. (2005) Separation of sperm and epithelial cells in a microfabricated device: potential application to forensic analysis of sexual assault evidence. Anal Chem 77: 742749.

19. Cho BS, Schuster T, Zhu X, Chang D (2002)A microfluidic device for separating motile sperm from nonmotile sperm via inter-streamline crossings. 2nd Annual Internaional IEEE-EMBS Special Topic Conference on Microtechnologies in Medicine \& Biology.

20. Lin YN, Chen PC, Wu RG, Pan LI (2013) Micro diffuser-type movement inversion sorter for high-efficient sperm sorting. Nano/Micro Engineered and Molecular Systems (NEMS), 2013 8th IEEE International Conference on 10581061.

21. Ainsworth C, Nixon B, Aitken RJ (2005) Development of a novel electrophoretic system for the isolation of human spermatozoa. Hum Reprod 20: 2261-2270.

22. Cho BS, Schuster TG, Zhu X, Chang D, Smith GD, et al. (2003) Passively driven integrated microfluidic system for separation of motile sperm. Anal Chem 75: $1671-1675$
23. Hyakutake T, Hashimoto Y, Yanase S, Matsuura K, Naruse K (2009) Application of a numerical simulation to improve the separation efficiency of a sperm sorter Biomed Microdevices 11: 25-33.

24. Wu JM, Chung Y, Belford KJ, Smith GD, Takayama S, et al. (2006) A surfacemodified sperm sorting device with long-term stability. Biomed Microdevices 8: $99-107$

25. Wu TL (2007) Laminar Stream Defined Passive Fluidic Device for Motile Sperm Separation. IEEE 1st Annual International Conference Nano/Molecular Medicine Engineering.

26. McCormack MC, McCallum S, Behr B (2006) A novel microfluidic device for male subfertility screening. J Urol 175: 2223-2227.

27. Brown M, Wittwer C (2000) Flow cytometry: principles and clinical applications in hematology. Clin Chem 46: 1221-1229.

28. Huang HY, Wu TL, Huang HR, Li CJ, Fu HT, et al. (2014) Isolation of motile spermatozoa with a microfluidic chip having a surface-modified microchannel. J Lab Autom 19: 91-99. 